\title{
Situación actual del sustrato náhuatl en el español de Nicaragua
}

\section{Current situation of the Nahuatl substrate in Nicaraguan Spanish}

\author{
Zobeyda Catalina Zamora Úbeda \\ Universidad Nacional Autónoma de Nicaragua, Managua \\ zobeyda.zamora@unan.edu.ni \\ https://orcid.org/0000-0003-4016-5929
}

\begin{abstract}
(c) (1) (3)
(C) UNAN-Managua

Recibido: mayo 2020. Aceptado: junio 2020

DOI https://doi.org/10.5377/rll.v6i1.10123
\end{abstract}

\section{RESUMEN}

En el español de Nicaragua el sustrato náhuatl se presenta como la característica lingüística, en el nivel léxico, más identitaria. La herencia lingüística que dejaron los grupos nahuas en la cultura, específicamente, en la gastronomía, en nombres de plantas, animales y topónimos como nombres de ríos, lagos, montañas y los nombres de ciudades y pueblos es una herencia que, en la actualidad continua vigente, pero que en la medida que pasa el tiempo está siendo absorbida por la variante dominante, esto es, el español estándar. Los cambios culturales y tecnológicos han provocado un desplazamiento de las palabras procedentes o de origen náhuatl, así palabras como: chicle (goma de mascar), chibola (canica), chapa (arete), zacate (hierba), entre otros, son términos de uso común en el español nicaragüense, sin embargo, estos cada vez más están siendo desplazados por los equivalentes en el español estándar.

En la presente ponencia presentaré datos que están relacionados con 6 investigaciones que evalúan la vitalidad léxica de las palabras procedentes de la lengua náhuatl frente a los vocablos del español general o estándar en la variante nicaragüense. El objetivo de esos estudios consistió en identificar la preferencia de uso de la herencia lingüística que dejaron los nahuas, por medio de sustrato, frente a términos del español general o estándar. Por tanto, expondré las consideraciones o preferencias que presentaron los hablantes que participaron en los distintos estudios.

Palabras clave: léxico, nahualt, nicaragüense, sustrato, lingüística

\section{SUMMARY}

In Nicaraguan Spanish, the Nahuatl substratum is presented as the linguistic characteristic, at the lexical level, the most identity. The linguistic inheritance left by the Nahua groups in culture, specifically in gastronomy, in names of plants, animals and place names such as names of rivers, lakes, mountains and the names of cities and towns is a heritage that, today continues in force, but that as time passes it is being absorbed by the dominant variant, that is, standard Spanish. The cultural and technological changes have caused a displacement of the words from or of Nahuatl 
origin, as well as words such as: chewing gum (chewing gum), chibola (marble), sheet metal (earring), grass (grass), among others, are terms of Commonly used in Nicaraguan Spanish, however, these are increasingly being displaced by equivalents in standard Spanish.

In this presentation, I will present data related to 6 investigations that evaluate the lexical vitality of words from the Nahuatl language against the words of general or standard Spanish in the Nicaraguan variant.
The objective of these studies was to identify the use preference of the linguistic inheritance left by the Nahuas, by means of substrate, compared to terms of general or standard Spanish. Therefore, I will present the considerations or preferences presented by the speakers who participated in the different studies.

Keywords: lexicon, nahualt, Nicaraguan, substrate, linguistics

\section{INTRODUCCIÓN}

Antes de profundizar en la situación actual del sustrato náhuatl, se aclara que en este estudio se utilizará la escritura de náhuatl en vez de nahua, esto siguiendo la referencia que data el Diccionario de la lengua española (DLE) debido a que en esta obra lexicográfica las definiciones se presentan en el lema o palabra náhuatl, por tanto, se infiere que es este último, es el término más usado por los hispanohablantes, aunque valga aclarar que las dos nomenclaturas se usan indistintamente por diferentes lingüistas que han abordado el tema (Matus, 2005; Arellano J., Arellano F. 2009; Quesada, 2009 y Herranz, 2017).

Nicaragua cuenta con una extraordinaria riqueza no solo cultural sino también lingüística. Su característica plurilingüe radica en el aporte lingüístico que le han dado varias lenguas indígenas (náhuatl, mangue, chorotega, matagalpa, miskito y sumo o mayagna) así como modernas (inglés, francés, italiano...).

El español de Nicaragua sienta sus bases, principalmente, en el náhuatl, lengua que proviene de la rama uto - azteca. Los pobladores que hablaban esa lengua vinieron a Nicaragua entre los años 1200 y 1250 a. C. cuando un grupo de esta población náhuatl se separó del grupo de los Pipiles que se habían establecido en El Salvador y se asentaron en Nicaragua en la costa del Pacífico y en la región de Rivas a quienes se les conoció con el nombre de nicaraos. Posterior a la conquista española se calcula que eran una población de aproximadamente 700000 habitantes distribuidos entre El Salvador, Guatemala y Nicaragua (Herranz, 2017, p. 11).

El grupo náhuatl fue la población que más se extendió antes de la época colonial. La lengua de este grupo ha sobrevivido en el español de Nicaragua como una huella lingüística en forma de sustrato. El término sustrato los define el Diccionario de la lengua española (DLE) desde el punto de vista lingüístico, como la lengua que ejerce una influencia en otra instalada posteriormente en el mismo territorio. Por su parte, Dubois (1979) lo define como: 
El sustrato designa a toda lengua hablada que, en una región determinada, ha sido sustituida por otra lengua por diversas razones, cuando se considera la influencia que ha podido tener la lengua anterior sobre la lengua que le ha sucedido (p. 594)

En el caso del español de Nicaragua se refiere a la in fluencia que dejó la lengua náhuatl sobre el español a partir de la colonia española. La variante lingüística nicaragüense, se caracteriza principalmente por la influencia o herencia lingüística de la lengua náhuatl, por ejemplo, Mántica (2008) afirma que el nombre del país proviene de esta lengua nic (aquí) y atInahuac (junto al agua) = Nicaragua (Aquí junto al agua).

La constante evolución de las culturas y los pueblos debido a los avances tecnológicos (redes sociales, la televisión, la radio, el cine y la música) inciden en los cambios y giros de una lengua y eso hace que esta no sea estática, es decir, en la medida que las culturas y pueblos evolucionan lo hacen también las lenguas. En las últimas dos décadas los avances en estos ámbitos han proliferado de tal manera que han llegado a poner en peligro la herencia lingüística que dejaron distintos grupos indígenas y que hoy en día constituyen los llamados regionalismos, es decir, los vocablo o giro privativo de una región determinada o palabras propias de un país o región frente al español estándar. Esos cambios culturales han incidido en el idiolecto (conjunto de rasgos propios que emplea un dividuo al expresarse) de los hablantes, sobre todo en los jóvenes, quienes tienen preferencia, cada vez más, por la utilización de las palabras del español general o estándar en lugar de las de procedencia indígenas, en nuestro caso, el náhuatl.

Frente a estos cambios inevitables, ha surgido una inquietud de mi parte indagar la vitalidad léxica de las voces de origen náhuatl, lo cual me ha llevado plantearme interrogantes como: ¿Será que las 600 voces que presenta Mántica (2008) en su Breve diccionario de voces náhuatl en la obra El habla nicaragüense y otros ensayos están vigentes aún en el habla popular del nicaragüense? ¿El español general o estándar estará absorbiendo las voces de origen náhuatl? ¿Consideran los hablantes nicaragüenses las voces náhuatl como parte de su identidad? ¿Tendrán conciencia los nicaragüenses que a diario utilizan las voces de origen náhuatl? Las respuestas a esta y otras preguntas me ha inducido a realizar una aventura exploratoria, la cual no sería posible si no contara con la colaboración de alumnos y exalumnos (Jara y Orozco, 2015; Cortez y Cruz, 2015; Rostrán y Quintanilla, 2015; Largaespada y Delgado, 2016: Luna, J. y Luna, 2016 y Martínez y Flores, 2017) de las carreras de Filología y Comunicación, y Lengua y Literatura de la Universidad Nacional Autónoma de Nicaragua (UNAN - Managua) junto a quienes me he embarcado a investigar la vigencia o vitalidad que tienen los nahuatlismos en el español en las distintos lugares del país, principalmente en el Pacífico de Nicaragua (Masaya, León y Managua) zona en la que se establecieron los náhuatl. 


\section{METODOLOGÍA}

En las investigaciones realizadas se utilizó una metodología cuantitativa, la primera parte consistió en nombrar objetos representados en imágenes cuyos nombres se podrían mencionar en náhuatl o español estándar, por ejemplo, se presentó la imagen de un biberón/pacha (término usado en el español de Nicaragua) y se les preguntó a los informantes el nombre representado en la imagen. En la segunda parte se realizaron preguntas incompletas para que los encuestados las completaran; por ejemplo, ¿A la fruta que no ha sazonado se le llama? Los informantes contestaron: verde, tierno y el nahuatlismo celeque. La tercera parte, se relacionó con las actitudes lingüísticas de los hablantes frente a la preferencia entre léxico del español estándar y el náhuatl, por ejemplo, se les preguntó ¿Si les gustaría que en la enseñanza secundaria se les instruyera en la procedencia de las palabras náhuatl? ¿Si conocían palabras de origen náhuatl? Que las mencionaran, entre otras.

Por otro lado, valga decir que, aunque los náhuatl o nicaraos se asentaron en la zona del Pacífico, las palabras no son de uso exclusivo solo en esta región, debido a que las palabras no tienen fronteras, emigran con sus hablantes, un ejemplo al respecto, son los nahuatlismos en Costa Rica, país en el que no llegaron a establecerse los náhuatl en tiempo de la colonia en el Valle Central, Según Quesada:

La conquista del Valle Central fue organizada por grupos de exploradores y colonos provenientes de América Central, en cuya habla ya estaban arraigadas muchísimas palabras de origen azteca. Gran cantidad de esas voces lograron pasar a Costa Rica; algunas de estas son: achote, agüizote, atol, ayote, caite camote, chapulín, chayote, chile, entre otras." (Quesada, 453).

Además, se tendría que añadir la importante migración de más de un millón de nicaragüenses que en los últimos treinta años se han establecidos en el vecino país debido a factores político y económicos, quienes, en su mayoría, llevan con ellos las particularidades lingüísticas o rasgos propios del español de Nicaragua y las tradiciones.

En los resultados de las investigaciones vinculadas con la vitalidad léxica del sustrato náhuatl realizadas por: Jara y Orozco (2015); Cortez y Cruz (2015); Rostrán y Quintanilla (2015); Largaespada y Delgado (2016): Luna, J. y Luna (2016) y Martínez y Flores (2017) para comprobar la vigencia o vitalidad del sustrato náhuatl se seleccionó un corpus o glosario del Breve diccionario de nahuatlismo de Mántica (2008) dejando de un lado los topónimos (nombres propios de lugares: ciudades, pueblos, montañas, lagos, lagunas y ríos) debido a que, en el proceso de la evolución léxica, no son nombres que puedan sufrir cambios tan fácilmente porque los hablantes los utilizan como parte de identificación con el lugar, así las toponimia ofrecen 
testimonio de la existencia de palabras que perduran más en el tiempo, pues sus formaciones permanecen de una generación a otra, es decir, en la mayoría de los casos, se encuentran fosilizados.

\section{RESULTADOS DE LAS INVESTIGACIONES}

Los estudios acerca de la vitalidad léxica evidenciaron que existe decadencia del sustrato náhuatl en el habla del nicaragüense. Así en el trabajo realizado por Cortez y Cruz (2015) los hablantes encuestados dejaron entrever la preferencia de los informantes hacia el español estándar, a pesar de que reconocieron la presencia de las voces náhuatl, los encuestados consideraron que al utilizar los términos de ese origen eran vistos como personas incultas. En este caso, se presentó un estigma hacia esas palabras debido a que no las consideraron prestigiosas.

Por otra parte, en el estudio realizado por Jara y Orozco (2015) Los resultados proyectaron que los informantes utilizan el léxico náhuatl, pero desconocen su procedencia; además, en lo relacionado con las actitudes lingüísticas que tienen los hablantes hacia esos términos se presentó cierta inseguridad lingüística, pues los hablantes consideraron como correctas y adecuadas las palabras del español estándar, pero prefieren utilizar en la cotidianidad las del sustrato náhuatl. Esto último concuerda con el estudio realizado por Zamora (2015) sobre las actitudes lingüísticas en Nicaragua, cuando afirma que los nicaragüenses sienten inseguridad sobre su variante dialectal al considerarla menos correcta que otras, pero muestran lealtad lingüística al preferir el español de Nicaragua por encima de otras variantes.

Asimismo, el estudio realizado por Largaespada y Delgado (2015) evidencia que los hablantes evalúan la herencia léxica náhuatl como correcta, sin embargo, los informantes manifestaron que las palabras de ese origen son incultas y vulgares, y afirmaron que su uso solo debe darse en contextos de confianza entre amigos y familiares. Sin embargo, nuevamente se presenta lealtad cuando expresaron que tienen interés de que en la escuela se les enseñe a distinguir las palabras de origen náhuatl.

Otros resultados de los estudios realizados muestran que la mayoría de los nicaragüenses desconocen las palabras utilizadas en la cotidianidad, principalmente las de la lengua indígena tal es el caso de: pacha (biberón), chibola (canica), chicle (goma de mascar) achiote (fruto y semilla de la bija), chayote, zacate (hierba), chiltoma (pimiento o chile dulce), entre otras. En los seis estudios realizados hasta este momento, se les solicitó a los hablantes que mencionaran palabras de procedencia náhuatl y la mayoría solo mencionó toponimias, nombre de lugares como: Coyotepe (cerro), Asososca (laguna), Diriamba (ciudad), Masatepe 
(ciudad), Xolotlán (lago), Managua (ciudad), entre otras. Según los informantes en la escuela solo se les enseña el origen de las toponimias náhuatl.

En líneas generales, en los resultados de las investigaciones se evidencia que la vitalidad léxica de algunos nahuatlismos estuvo marcada con menos del 50\% de uso, y que, por tanto indica que estos se utilizan cada vez menos por los hablantes, estos son: zonchinche (ave rapaz), chigüín (niño o adolescente), cacahuate (maní), chapulín (saltamonte), papalote (cometa), chingorro (gorro), chingo (corto), niste (desteñido), celeque (tierno), chimadura (herida causada en la piel) y chimar (molestar). Vale aclarar que estos términos fueron más utilizados por personas adultas, lo cual puede significar que dichas palabras puedan desaparecer, con el paso del tiempo, del idiolecto de los nicaragüenses una vez que estos hablantes lleguen a su término de vida y no las transmitan a las próximas generaciones.

Por su parte, mantienen vigencia con más del 60\% palabras como: chiltoma (pimiento o chile dulce), pacha (biberón), chapa (arete), chompipe (pavo), mecate (cordel o cuerda), elote (mazorca tierna de maíz), chayote (vegetal), y guacal/jícara (vasija hecha del guacal, fruto), chistata (cistitis o micción dolorosa), colochos (rizos), chingaste (residuos de los granos molidos o quebrados), cumiche (hijo menor en una familia), machigüe (desperdicios de comida), zopilote (ave rapaz), cuapes (mellizo), caite (sandalia) y chimpapo (persona que carece de uno o más dientes), ayote (vegetal), chacuatol (revoltijo de cosas), tequio (tarea que debe cumplirse con esmero) y alaste (viscoso).

Las palabras que mostraron peligro de extinción al obtener un uso por menor al 40\% son: apapachar (abrazar), cacaste (flaco), tilinte (estirado o persona muerta), mayate (pálido) y pepenar (recoger), chincaca (rabadilla de persona o de ave) y pipilacha (libélula), estas fueron mencionadas, principalmente, por personas mayores, por tanto, es muy probable que desaparezcan del léxico del español de Nicaragua.

Por otro lado, se han realizado cuatro investigaciones en el nivel educativo, así Francisca Rostrán y María Quintanilla (2015) realizaron un estudio sobre la vitalidad de las palabras de origen náhuatl en los estudiantes de secundaria del colegio Gaspar García Laviana, del municipio de Tipitapa, en este trabajo se evidenció que los estudiantes utilizan las palabras procedentes del náhuatl, sin embargo, la mayoría desconocen su origen. Daisy Largaespada y Raúl Delgado (2016) investigaron la Vitalidad léxica de los nahuatlismos en los docentes de Lengua y Literatura de los colegios públicos de Managua; en este estudio realizado a los docentes de educación secundaria se constata que, también, los profesores de la especialidad (Lengua y Literatura) desconocen que las palabras proceden de la lengua en cuestión.

Por su parte, tanto la investigación realizada por: Izayda Berríos, Karla Mojica y Tania Bermúdez (2019) La enseñanza del sustrato náhuatl en octavo grado de secundaria: una propuesta didáctica de como enseñar 
el sustrato náhuatl de una manera lúdica y el trabajo realizado por Diana Figueroa, José Carlos y Cristian Flores: Propuesta didáctica para la enseñanza de las voces náhuatl a través de la escritura de anécdotas en secundaria resaltaron que la mayoría de los estudiantes utilizan palabras del sustrato náhuatl en diferentes contextos: familia, escuela y entre amigos, sin embargo, los discentes consideraron esta variante léxica como poco prestigiosa y las valoraron como coloquial e inculta. Ambos estudios resaltan que los estudiantes no logran reconocer el léxico de procedencia náhuatl.

\section{CONCLUSIONES}

Los estudios realizados evidencian una necesidad de incluir con mayor profundidad el estudio del sustrato náhuatl en el sistema educativo de primaria y secundaria con el fin de afianzar un rasgo lingüístico que es parte de la identidad del nicaragüense, pues es precisamente en el sistema educativo donde se debe reforzar el valor hacia la variante nicaragüense, la cual debe de estar contextualizada con la enseñanza del español a nuestra realidad lingüística.

Muchos de las palabras de origen náhuatl están cayendo en desuso debido a que estas son estigmatización por parte de los hablantes por ser palabras que tienen su origen en una lengua indígena. Los estudios vinculados con la vitalidad léxica del sustrato náhuatl mostraron que muchos de los hablantes nicaragüenses encuestados tienen inseguridad lingüística cuando los informantes consideran esa herencia lingüística como términos inadecuados, sin embargo, aceptan o sugieren que en la enseñanza escolar se les instruya a identificarlos, hecho que concuerda con el estudio realizado por Zamora (2015) sobre Las actitudes lingüísticas en Nicaragua.

Por otro lado, en las investigaciones se comprueba la teoría de Mántica quien afirma que: "e I náhuatl se oculta, pero no desaparece. Se disfraza de palabras castellanas para poder sobrevivir, pero sigue siendo náhuatl (Mántica, 2008, p. 107). Esto quiere decir que en Nicaragua existe un náhuatl oculto, es decir, los hablantes tienen desconocimiento acerca de la procedencia de los términos provenientes de dicha lengua indígena, sin embargo, los utilizan en la cotidianidad con mucha frecuencia.

Los resultados de los estudios aquí mencionados muestran que la enseñanza en nivel del léxico, en la educación de primaria y secundaria en Nicaragua no está contextualizada según la variante lingüística del dialecto del español hablado en el país, pues en los libros de texto de la signatura de Lengua y Literatura, orientados por el Ministerio de Educación, solamente en octavo grado se menciona como tema las toponimias indígenas nicaragüenses entre las que destacan, principalmente, las de origen náhuatl. Por tanto, la enseñanza del rasgo más característico a nivel léxico de la variante del español es casi nula, hecho 
que ha llevado a que los hablantes solo tengan como referente de los orígenes de la lengua náhuatl las toponimias, por consiguiente, esto parece ser la causa de la preferencia de las palabras del español estándar por encima de la herencia lingüística náhuatl.

\section{REFERENCIAS}

Arellano, F. (2009) Diccionario del Español de Nicaragua (DEN), PAVSA, S. A. Managua, Nicaragua.

Arellano, J. E. (ed.). (1992) El Español de Nicaragua. Managua, Instituto Nicaragüense de Cultura Hispánica.

Figueroa, D., Flores, C. y Vásquez, J. C. (2019) Propuesta didáctica para la enseñanza de las voces náhuatl a través de la escritura de anécdotas en secundaria (Monografía para optar al título de licenciatura). UNAN - Managua.

Jara Gómez, J. y Orozco Palacio, M. (2015) La vitalidad léxica de los nahuatlismos en los hablantes del municipio de Cárdenas, Rivas: un enfoque sociolingüístico. (Monografía para optar al título de licenciatura). UNAN - Managua.

Herranza, A. (2017) Proceso de nahuatlización y nahuatlismos de uso en Honduras. Tegucigalpa, Honduras. Editorial Universitaria Universidad Nacional Autónoma de Honduras.

Largaespada Tijerino, D., y Delgado Lovo, R. (2016) Vitalidad léxica de los nahuatlismos en los docentes de Lengua y Literatura de los colegios públicos de Managua: un estudio sociolingüístico. (Trabajo de fin de curso de la asignatura Español de Nicaragua, inédito. UNAN - Managua.

Largaespada Tijerino, D., y Delgado Lovo, R. (2016) Análisis de la vitalidad léxica del sustrato náhuatl en los hablantes del municipio de Jinotepe, Nicaragua: un enfoque sociolingüístico (Monografía para optar al título de licenciatura) UNAN - Managua.

Luna Martínez, Y. y Luna Castillo Y. (2016) La vitalidad léxica de los nahuatlismos en los hablantes del municipio de Nagarote, León: un enfoque sociolingüístico (Monografía para optar al título de licenciatura) UNAN - Managua.

Mántica, C. (1998) El habla nicaragüense y otros ensayos. Managua: Hispamer.

Martínez, X. y Flores, J. C. (2017) Estudio de la vitalidad léxica del sustrato náhuatl en los hablantes del barrio Monimbó de Masaya: un enfoque sociolingüístico (Monografía para optar al título de licenciatura). UNAN - Managua. 
Rosales Solís, M. A. y Zamora Úbeda, Z. (2016) Diccionario de centroamericanismos (D/CA). Managua: Complejo Gráfico TMC.

Rostrán Miranda, F. y Quintanilla Rayo, M. (2015) Vitalidad de las palabras de origen náhuatl en los estudiantes de secundaria del colegio Gaspar García Laviana del municipio de Tipitapa. (trabajo de fin de curso, inédito) Managua: UNAN-Managua.

Quesada, M. A. (2009) Historia de la lengua española en Costa Rica. San José, C. R. Editorial UCR.

Zamora Úbeda, Z. (2015) Las actitudes lingüísticas en Nicaragua: lo que pensamos los nicaragüenses sobre el español que hablamos. Managua: PAVSA 